\title{
Funcionalidade e qualidade de vida de pessoas com esclerose lateral amiotrófica e percepção da sobrecarga e apoio social de cuidadores informais
}

\section{Functionality and quality of life of people with amyotrophic lateral sclerosis and perception of overload and social support of informal caregivers}

\author{
(D) Crystian Moraes Silva Gomes ${ }^{1}$, (D) Aline Caus Zuqui ${ }^{1}$, (Dellen Valladão Schiavo ${ }^{1}$, IJonaina Fiorim Pereira de Oliveira ${ }^{1}$
}

${ }^{1}$ Centro de Reabilitação Física do Espírito Santo - CREFES

Correspondência

Crystian Moraes Silva Gomes

E-mail: crystian salazar@hotmail.com

Submetido: 07 Agosto 2020

Aceito: 21 Outubro 2020

Como citar

Gomes CMS, Zuqui AC, Schiavo KV, Oliveira JFP. Funcionalidade e qualidade de vida de pessoas com esclerose lateral amiotrófica e percepção da sobrecarga e apoio social de cuidadores informais. Acta Fisiatr. 2020;27(3):166-173.

DOI: 10.11606/issn.2317-0190.v27i3a172216

\begin{abstract}
RESUMO
Objetivo: Esta pesquisa tem como objetivo mensurar a funcionalidade e qualidade de vida de pacientes com Esclerose Lateral Amiotrófica (ELA) e fornecer evidências sobre a possível sobrecarga de cuidados gerados aos seus cuidadores informais. Método: Tratase de um estudo descritivo exploratório do tipo quantitativo, com delineamento transversal. A amostra do estudo foi composta por 24 participantes, sendo 12 pacientes com ELA e 12 cuidadores informais, recrutados em um Centro Especializado de Reabilitação. Foram administrados aos indivíduos com ELA os instrumentos: Questionário sobre Informações Demográficas; Amyotrophic Lateral Sclerosis Functional Rating Scale-Revised (ALSFRS-R); Amyotrophic Lateral Sclerosis Assessment Questionnaire (ALSAQ-40) e aos cuidadores: Questionário de Avaliação da Sobrecarga do Cuidador Informal (QASCI); Escala de Apoio Social do Medical Outcomes Study (MOS). Resultados: Foram encontradas fortes correlações entre ALSFRS-R e ALSAQ-40 ( $r=-0.709$ e $p<0.010)$, entre o domínio Atividades de Vida Diária e a ALSFRS-R ( $r=-0.877$ e $p<.001)$, entre os domínios da QASCl e MOS, Mecanismo de Eficácia e de Controle e Apoio material ( $r=-0.598$ e $p<0.040$ ), Satisfação com o Papel e com o Familiar e Apoio Afetivo ( $r=-0.604$ e $p<0.037)$, e Suporte Familiar e Interação Social Positiva ( $r=-0.683$ e $p<$ 0.014). Conclusão: A funcionalidade e a qualidade de vida do paciente com ELA influenciam na provisão de cuidados, o suporte social percebido foi uma variável moderadora da sobrecarga de estresse dos cuidadores.
\end{abstract}

Palavras-chave: Esclerose Amiotrófica Lateral, Qualidade de Vida, Cuidadores, Apoio Social, Centros de Reabilitação

\section{ABSTRACT}

Objective: This research aims to measure the functionality and quality of life of Amyotrophic Lateral Sclerosis (ALS) patients and provide evidence about the potential excessive burden of care generated for their informal caregivers. Method: This is a quantitative, exploratory and descriptive study, and cross sectional investigation. The study sample was 24 participants, being 12 patients with ALS and 12 informal caregivers, recruited at a Specialized Rehabilitation Center. Individuals with ALS were administered the following instruments: Demographic Information Questionnaire; Amyotrophic Lateral Sclerosis Functional Rating Scale-Revised (ALSFRS-R); Amyotrophic Lateral Sclerosis Assessment Questionnaire (ALSAQ-40) and caregivers: Informal Caregiver Burden Assessment Questionnaire (QASCI); Medical Outcomes Study Social Support Survey (MOS). Results: Strong correlations were found between ALSFRS-R and ALSAQ-40 $(r=-0.709 p<0.010)$, between the Daily Living Activities domain and ALSFRS-R $(r=-0.877$ $\mathrm{p}<.001$ ), between the domains of QASCl and MOS, Efficiency and Control Mechanism and material support $(r=-0.598 p<0.040)$, Role and Family Satisfaction and Affective Support ( $r=-0.604 p<0.037$ ), and Family Support and Interaction Positive Social ( $r=-$ $0.683 p<0.014)$. Conclusion: The functionality and quality of life of the patient with ALS influence the provision of care, the perceived social support was a moderating variable of the caregivers' stress burden.

Keywords: Amyotrophic Lateral Sclerosis, Quality of Life, Caregivers, Social Support, Rehabilitation Centers 


\section{INTRODUÇÃO}

A Esclerose Lateral Amiotrófica (ELA) é uma doença neurodegenerativa e progressiva associada à perda de neurônios motores superiores e inferiores. É caracterizada por fraqueza da musculatura esquelética, com declínio progressivo da capacidade funcional, acompanhada de disartria, disfagia e falência da musculatura respiratória. ${ }^{1-3}$ Nessa doença, não há comprometimento primário das funções sensitivas, vésicoesfincterianas e sexuais como também das funções cognitivas. ${ }^{2,3}$

A etiologia da ELA é multifatorial, incluindo componentes genéticos e ambientais. ${ }^{3,4}$ Estudos recentes relatam que esta doença é mais comum em sujeitos do sexo masculino e a taxa de sobrevida média dos pacientes de 3 a 5 anos, sendo a insuficiência respiratória a maior causa de mortalidade. ${ }^{1,2,4}$

Todavia, $10 \%$ dos casos apresentam uma forma de progressão da doença mais lenta e podem atingir uma sobrevida de mais de uma década. ${ }^{2} \mathrm{~A}$ velocidade variável de progressão da ELA e a sua apresentação clínica heterogênea tornam seu diagnóstico e manejo clínico desafiadores. Geralmente, o declínio funcional se inicia nas extremidades, em especial nos membros superiores, progredindo posteriormente para membros inferiores, tronco, musculatura faríngea e respiratória. ${ }^{5}$

Os sinais e sintomas clínicos supracitados culminam na incapacidade do indivíduo com ELA para realizar suas atividades de vida diárias (AVD's), e podem levar a disfagia e a insuficiência respiratória. Adicionalmente, a severidade da doença e as incertezas quanto ao curso, o agravamento de incapacidades e a evolução da carga de cuidados, são causa de grande estresse para os indivíduos afetados e suas famílias. ${ }^{1,2,5}$

O manejo clínico dos indivíduos com diagnóstico de ELA é complexo e requer uma abordagem abrangente e multidisciplinar. Os objetivos gerais do cuidado são maximizar o desempenho funcional e melhorar a qualidade de vida. 0 foco do cuidado pode mudar à medida que a doença progride, assim entender a progressão da doença permite aos profissionais envolvidos no processo de reabilitação se antecipar às mudanças nas prioridades de atendimento $e$ prevenir possíveis complicações. ${ }^{1,2,4,5}$

À medida que a ELA progride, as pessoas acometidas aumentam o grau de incapacidade, solicitando maior assistência e recursos externos de apoio, em geral estes são fornecidos por um cuidador principal, que assume a responsabilidade de cuidar durante todo o processo da doença. ${ }^{6} \mathrm{O}$ acompanhamento da pessoa com ELA implica em uma reorganização familiar, onde algum membro assume a função do cuidado, executando atividades que podem ser esporádicas ou permanecentes.

O cuidar, mais do que um ato, é uma atitude, pois abrange um momento de atenção e zelo e constitui uma atividade de ocupação, responsabilização e envolvimento afetivo com o outro. $^{5}$

É considerado cuidador informal a pessoa não remunerada, familiar ou amiga, que assume o papel de principal responsável pela organização ou assistência e cuidados prestados à uma pessoa dependente. ${ }^{7}$ Esta forma de prestação de cuidados ocorre habitualmente no domicílio do interveniente. A coabitação facilita a prestação de cuidados, sendo mais frequente quando os cuidadores são os cônjuges ou os descendentes.

Se faz imprescindível ressaltar, que cuidar de pessoas com ELA é extremamente exigente tanto para a pessoa cuidada como para a família e os cuidadores, pois esta patologia envolve um número vasto de alterações e incapacidades funcionais, questões emocionais e psicossociais, além da possibilidade de situações de dependência total. A doença muda drasticamente o curso da vida de todas as pessoas envolvidas, direta ou indiretamente. ${ }^{1}$

Os cuidadores devem fazer parte do programa de cuidados da pessoa com ELA, a fim de criar estratégias para apoiar e garantir o bem-estar dessa população. ${ }^{8}$ Profissionais precisam considerar a experiência do cuidador quando serviços de assistência e reabilitação são planejados, sendo importante adotar cuidados centrados, não apenas na pessoa com ELA, mas também para seus cuidadores informais, pois fatores relacionados a ambas as partes são associados a percepção sobre a satisfação e a qualidade de vida. ${ }^{4}$

A manutenção das redes sociais torna-se relevante para os indivíduos com ELA e seus cuidadores. O cuidado constante associado às relações afetivas faz da depressão e da ansiedade um evento comum entre cuidadores. ${ }^{9}$ A redução destas redes está intimamente ligada a solidão e ao isolamento, dificultando a integração com outras pessoas e/ou grupos em suas comunidades, limitando a comunicação e a manutenção de contatos sociais mínimos.

O apoio social, pode ser considerado como os recursos postos a disposição por outras pessoas em situações de necessidade. ${ }^{10}$ Uma rede de apoio funcional, auxilia a lidar com situações estressantes advindas do papel de cuidar e na reorganização do cotidiano.

$\mathrm{O}$ ato de cuidar de um paciente com ELA, durante o tempo de manifestação da doença, nos faz refletir sobre a necessidade e a forma de avaliar a sobrecarga do cuidador e o apoio social percebido. A avaliação da sobrecarga do cuidador através de instrumentos específicos permite identificar as fragilidades e criar estratégias para redução de estresse e diminuição de sobrecarga que podem ser causadas ao cuidar do outro. ${ }^{5}$

Todavia, a avaliação do apoio social, além de indicar o quão integrado socialmente é o indivíduo, indica o grau com que as relações interpessoais correspondem a determinadas funções e como oferecem suporte em momentos de crise ou readaptação. ${ }^{10}$

No Brasil, ainda são escassas na literatura, pesquisas a respeito da percepção da sobrecarga e do apoio social em familiares/cuidadores informais, e a relação com a funcionalidade e qualidade de vida do indivíduo com Esclerose Lateral Amiotrófica. Nesse sentido, esta pesquisa se faz relevante, visto a eminente possibilidade de subsidiar aos profissionais de saúde o desenvolvimento de políticas, serviços e abordagens centrados na pessoa com ELA, na sua família e nos seus cuidadores.

\section{OBJETIVOS}

Mensurar a capacidade funcional e qualidade de vida de pacientes com Esclerose Lateral Amiotrófica, e a percepção da sobrecarga e o apoio social percebido pelos seus cuidadores informais. 


\section{MÉTODO}

Trata-se de um estudo descritivo exploratório do tipo quantitativo, com delineamento transversal. Este estudo foi aprovado pelo Comitê de Ética em Pesquisa do Centro de Ciências da Saúde da Universidade Federal do Espírito, recebendo o parecer de número 3.395.238 de 2019 (CAEE:10702919.7.0000.5060). Todas as etapas da pesquisa seguiram as orientações da Resolução n 466/2012 e Resolução n $510 / 2016$ do Conselho Nacional de Saúde.

A amostra deste estudo foi composta por 24 participantes, sendo 12 pacientes com diagnóstico de Esclerose Lateral Amiotrófica e 12 familiares/cuidadores, acompanhados na Unidade de Tratamento Neurológico Adulto do Centro de Reabilitação Física do Espírito Santo (CREFES), localizado em Vila Velha - Espírito Santo.

Foram incluídos, neste estudo, pacientes nos diversos estágios da ELA, sendo os participantes selecionados no período compreendido entre setembro a novembro de 2019.

Foi adotado como critérios de inclusão dos pacientes: ambos os sexos; ausência de outras alterações neurológicas e/ou outras doenças sistêmicas associadas; diagnóstico de ELA; idade igual ou superior a 18 anos; capacidade de comunicação verbal ou efetiva através de recursos de comunicação assistiva, ou por meio de acompanhantes que possam servir de intermediários para confirmar a aceitação e participação no estudo.

Os critérios de inclusão dos familiares/cuidadores foram: ser o principal familiar/cuidador do paciente assistido com diagnóstico de Esclerose Lateral Amiotrófica; ter idade igual ou superior a 18 anos; estar disponível para o estudo e de acordo com as exigências da pesquisa. Foram excluídos os familiares/cuidadores de pacientes com patologias associadas que pudessem influenciar no resultado do estudo, aqueles que recebem remuneração pela tarefa de cuidar e com formação acadêmica na área da Saúde.

Os indivíduos foram convidados a participar da pesquisa por intermédio dos profissionais de reabilitação do CREFES. Os participantes desta pesquisa foram avaliados por meio da administração de questionários e avaliações padronizadas, sendo este processo conduzido em formato de entrevista individual pelos autores do trabalho, que possuem graduação em Terapia Ocupacional e Fisioterapia com ampla experiência clínica na avaliação de processos de funcionalidade e incapacidade humana.

\section{Instrumentos de Avaliação}

Questionário sobre Informações Demográficas; foi administrado um questionário para a descrição amostral no que se refere às características sociodemográficas. O nível socioeconômico dos participantes foi avaliado por meio do Critério de Classificação Econômica Brasil, proposto pela Associação Brasileira de Empresas de Pesquisa. ${ }^{11}$

A aplicação deste questionário tem por objetivo classificar as classes econômicas dos participantes, em um dos seis estratos socioeconômicos denominados A, B1, B2, C1, C2 e D$\mathrm{E}$.

Amyotrophic Lateral Sclerosis Functional Rating ScaleRevised (ALSFRS-R); é um instrumento específico para mensuração da funcionalidade de pacientes com ELA, sendo validado e adaptado transculturalmente para a língua portuguesa. ${ }^{12}$

A ALSFRS-R/BR consiste em 12 itens (Fala, Salivação, Deglutição, Escrita, Cortando Alimentos, Vestuário e Higiene, Transferências, Andar, Subir Escadas, Dispnéia, Ortopnéia e Insuficiência Respiratória), com escore de zero a quatro em cada item, e um escore total variando entre 0 a 48 pontos, onde 48 pontos significam funcionalidade normal e 0 séria incapacidade.

Amyotrophic Lateral Sclerosis Assessment Questionnaire (ALSAQ-40); foi utilizado para caracterizar a qualidade de vida dos indivíduos com ELA. Trata-se de um questionário de qualidade de vida específico para essa população, com adaptação transcultural para a população brasileira. ${ }^{13}$

O ALSAQ-40 consta com 40 questões divididas em cinco dimensões: Atividades de Vida Diária e Independência (10 itens), Aspectos Físicos (10 itens), Alimentação (3 itens), Comunicação (7 itens) e Aspectos Emocionais (10 itens). Para cada item há cinco possibilidades de resposta: "nunca", "raramente", "às vezes", "frequentemente" e "sempre". A pontuação total e de cada domínio da escala varia entre 0 e 100. Uma pontuação de 0 a 19 em um domínio significa que o paciente não apresenta dificuldade; 20 a 39, raramente apresenta dificuldade; 40 a 59, às vezes apresenta dificuldade; 60 a 79, frequentemente apresenta dificuldade; e 80 a 100 sempre apresenta dificuldade.

Questionário de Avaliação da Sobrecarga do Cuidador Informal (QASCI); Instrumento adaptado e com validação transcultural para a população brasileira. ${ }^{7}$ É composto por 32 itens, que integram sete dimensões: Implicações na vida pessoal (11 itens); Reações às exigências (5 itens); Sobrecarga emocional (4 itens); Sobrecarga financeira (2 itens) e Mecanismos de eficácia e de controle (3 itens); Suporte familiar (2 itens); Satisfação com o papel e com o familiar (5 itens). Cada item é avaliado por uma escala ordinal de frequência que varia de um a cinco categorias de respostas: "não/nunca", "raramente", "às vezes", "quase sempre", "sempre".

O escore final resulta da soma total das respostas obtidas para os 32 itens (intervalo possível de 32 a 160), após a inversão das pontuações dos itens das três dimensões positivas. Mecanismo de eficácia e de controle, Suporte familiar e Satisfação com o papel e com o familiar, constituem forças positivas na dinâmica do cuidado, isto é, diminuem o resultado da sobrecarga. Um escore total mais altos corresponde a situações com maior peso ou maior sobrecarga.

Escala de Apoio Social do Medical Outcomes Study (MOS); A Escala de Apoio Social do Medical Outcomes Study, foi desenvolvida para mensuração do apoio social em pacientes com doenças crônicas e abrange 19 itens, distribuídos em 4 dimensões de apoio social: Material, Afetivo, Emocional/Informação e Interação social positiva. Estudos brasileiros de validação e adaptação transcultural, desenvolvidos com diferentes grupos amostrais avaliaram a pertinência do modelo fatorial composto por quatro fatores. ${ }^{10}$

Para cada item do instrumento, indica-se a frequência que ocorre em cada tipo de apoio, numa escala tipo likert de cinco pontos. Os itens neste instrumento não especificam a fonte de apoio (p. ex., família, amigos, comunidade ou outros) e medem a percepção da disponibilidade de apoio funcional. O intervalo 
possível da pontuação total varia entre 19 e 95, sendo que quanto mais alto o escore, maior é o apoio social percebido.

Todas as análises foram desenvolvidas utilizando-se o software estatístico JAMOVI. ${ }^{14}$ Os dados atenderam aos pressupostos teóricos exigidos para o emprego de abordagem paramétrica, mensurado através do teste de Shapiro-Wilk.

Foram empregadas técnicas de análises estatísticas descritivas com índices de frequência, tendência central (média) e dispersão (desvio padrão) para a caracterização da amostra. Para as análises de correlação entre as variáveis, foi utilizado o coeficiente de pearson. As correlações foram classificadas como: muito fraca de 0,00 a 0,19 ; fraca de 0,20 a 0,39; moderada de 0,40 a 0,59; forte de 0,60 a 0,79 ; e muito forte de 0,80 a 1,00. Foi adotado neste estudo o nível de significância de $p<0.05$.

\section{RESULTADOS}

Participaram do estudo 24 sujeitos, sendo 12 pacientes com Esclerose Lateral Amiotrófica e 12 familiares/cuidadores informais. Sobre a caracterização dos pacientes, 6 correspondem ao sexo masculino (50\%) e 6 ao sexo feminino (50\%). Em relação ao estado civil, 8 são casados (66.7\%), 2 são divorciados (16.7\%), 1 separado e 1 solteiro, ambos com $8.3 \%$.

O nível de escolaridade dos pacientes variou entre $5 \mathrm{com}$ Ensino Superior (41.7\%), 4 com Ensino Médio Completo (33.3\%), 1 Não Alfabetizado, 1 com Ensino Fundamental Incompleto e 1 com Ensino Médio Incompleto, todos com 8.3\% cada. A média de idade dos pacientes foi de 61 anos. 0 tempo de diagnóstico médico obteve média de 28.3 meses. Em relação a gravidade da doença os participantes foram classificados em quatro níveis: ${ }^{15}$ leve (25\%), moderado (8.3\%), grave $(41.6 \%)$, terminal $(25 \%)$.

Neste Centro Especializado de Reabilitação o tramento multidisciplinar do paciente com ELA possui três modalidades, sendo a amostra costituida por $25 \%$ em estágio inicial de tratamento, $58.3 \%$ em estágio de acompanhamento de reabilitação, e $16.6 \%$ em estágio de monitoramento, readpatação e cuidados paliativos. 0 tempo de acompanhamento no serviço obteve média de 13.3 meses.
Sobre os Cuidadores, 8 (63.7\%) correspondem ao sexo feminino, e 4 (33.3\%) ao masculino. O grau de parentesco com o familiar com ELA corresponde, a 4 esposas (33.3\%), 3 irmãos e 3 filhos com $25.0 \%$ cada, e 2 maridos (16.7\%). A classe econômica familiar dos participantes foi classificada através do CCEB como, B2 (33.3\%), B1 (25.0\%), A (16.7\%), C1 (16.7\%), C2 (8.3\%).

Verifica-se através dos resultados da análise estatística do instrumento ALSFRS-R declínio na funcionalidade, apresentando média de 28.5 pontos. Todos os domínios da ALSAQ-40/BR nos 12 pacientes avaliados foram afetados pelo curso da doença (Tabela 1).

Observa-se que a pontuação média obtida no questionário ALSAQ-40/BR foi de 47.2 pontos, sendo que os domínios Atividade de Vida Diária (72.7 pontos) e Mobilidade (70.2 pontos) foram os mais afetados, ocasionando impacto na qualidade de vida dos pacientes com Esclerose Lateral Amiotrófica.

$\mathrm{Na}$ análise descritiva da QASCl, constatamos uma média total de 68.0 pontos, indicando sobrecarga moderada dos familiares/cuidadores informais. Os domínios mais afetados pelo papel de cuidador foram: Implicações na vida pessoal (26.0 pontos) e Reações a exigências (10.8 pontos).

Os fatores Mecanismo de eficácia e de controle (5.83 pontos), Suporte familiar (3.75 pontos) e Satisfação com o papel e com o familiar (7.42 pontos), constituem forças positivas na dinâmica do cuidado, isto é, os valores mais baixos encontrados nestas dimensões diminuem o resultado da sobrecarga.

O Apoio Social percebido, mensurado pela MOS demonstrou alta funcionalidade, com exceção do Apoio Emocional/Informação que apresentou percepção moderada (49.1 pontos).

As análises de correlações entre as variáveis podem ser observadas da Tabela 2. Constatamos correlações negativas fortes existentes entre o total ALSFRS-R e da ALSAQ-40. Obtivemos correlações negativas de muito forte intensidade entre a ALSFRS-R e o domínio Atividades de Vida Diária da ALSAQ-40.

Tabela 1. Análise dos instrumentos de avaliação

\begin{tabular}{|c|c|c|c|c|c|}
\hline Instrumentos & $\mathbf{N}$ & Média & Desvio Padrão & Mínimo & Máximo \\
\hline ASLFR-R & 12 & 28.5 & 25.5 & 15 & 44 \\
\hline ALSAQ-40 & 12 & 47.2 & 18.4 & 13.5 & 81.2 \\
\hline Mobilidade Física & 12 & 70.2 & 29.6 & 22.5 & 100 \\
\hline Atividades de Vida Diária & 12 & 72.7 & 31.8 & 5 & 100 \\
\hline Comer e Beber & 12 & 20.8 & 24.7 & 0 & 66.6 \\
\hline Comunicação & 12 & 44.3 & 35.4 & 0 & 100 \\
\hline Reações Emocionais & 12 & 46.0 & 22.5 & 10 & 100 \\
\hline QASCI & 12 & 68.0 & 21.8 & 45 & 122 \\
\hline Sobrecarga emocional & 12 & 9.08 & 3.48 & 5 & 17 \\
\hline Implicações na vida pessoal & 12 & 26.0 & 11.4 & 11 & 50 \\
\hline Sobrecarga financeira & 12 & 5.17 & 2.55 & 2 & 9 \\
\hline Reações a exigências & 12 & 10.8 & 5.58 & 5 & 25 \\
\hline Mecanismo de eficácia e de controle & 12 & 5.83 & 2.55 & 3 & 9 \\
\hline Suporte familiar & 12 & 3.75 & 2.45 & 2 & 10 \\
\hline Satisfação com o papel e com o familiar & 12 & 7.42 & 3.58 & 5 & 16 \\
\hline MOS & 12 & 74.6 & 14.1 & 51 & 94 \\
\hline Apoio Material & 12 & 67.9 & 22 & 40 & 100 \\
\hline Apoio Afetivo & 12 & 91.4 & 8.70 & 73 & 100 \\
\hline Apoio Emocional/ Informação & 12 & 49.1 & 15.6 & 16 & 70 \\
\hline Interação Social Positiva & 12 & 77.1 & 13.2 & 60 & 100 \\
\hline
\end{tabular}


Os valores totais da QASCl e da MOS não apresentaram correlações significativas entre si. Entretanto, nos domínios dos instrumentos confirmamos correlações negativas moderadas, entre Mecanismo de eficácia e de controle e Apoio material, e fortes entre Satisfação com o papel e com o familiar e Apoio Afetivo, e entre Suporte familiar e Interação social positiva.

Tabela 2. Análises de correlações entre variáveis

\begin{tabular}{lcc}
\hline & $\mathbf{R}$ & $\mathbf{P}$ \\
\hline ALSFRS-R X ALSAQ-40 & $-0.709^{* *}$ & 0.010 \\
ALSFRS-R X Atividades de Vida Diária & $-0.877^{* * *}$ & $<.001$ \\
QASCI X MOS & -0.076 & 0.815 \\
Mecanismo de Eficácia e de Controle X & $-0.598^{*}$ & 0.040 \\
Apoio Material & & \\
Satisfação com o Papel e com o Familiar X & $-0.604^{*}$ & 0.037 \\
Apoio Afetivo & $-0.683^{*}$ & 0.014 \\
Suporte Familiar X Interação Social Positiva & - &
\end{tabular}

\section{DISCUSSÃO}

Este estudo teve como objetivo mensurar a capacidade funcional e qualidade de vida de pacientes com diagnóstico de Esclerose Lateral Amiotrófica, e a percepção da sobrecarga de cuidados e apoio social percebido pelos seus cuidadores informais. A reabilitação multidisciplinar do paciente com ELA deve ser instituída no estágio inicial da doença e ser abordada de maneira orientada aos problemas inerentes a cada fase da doença, para manutenção do desempenho funcional e da qualidade de vida do indivíduo. ${ }^{1}$ Apesar da complexidade da doença para o paciente e a família, poucos estudos são encontrados na literatura contendo avaliações concomitantes da qualidade de vida e funcionalidade dos pacientes com ELA. ${ }^{3}$

Embora nossos achados demonstrem uma realidade local, exploramos simultaneamente aspectos psicossociais importantes do paciente e do cuidador durante o acompanhamento de reabilitação, desenvolvendo uma abordagem centrada na pessoa e na família.

Nosso estudo mostrou uma forte correlação negativa entre a ALSFRS-R e ALSAQ-40, isso ocorreu devido ao sistema invertido de pontuação de um dos instrumentos. Essa correlação na verdade, indica que quanto maior os escore de funcionalidade, menor o grau de dificuldade nos itens da qualidade de vida. A Esclerose Lateral Amiotrófica leva ao declínio da capacidade funcional, gerando grande impacto na qualidade de vida, sendo está diretamente relacionada à integridade física e independência funcional. ${ }^{3}$

Em nossa amostra, os domínios Atividades de Vida Diária e Mobilidade Física da ALSAQ-40 foram os que mais afetaram a qualidade de vida do indivíduo com ELA, entretanto, somente o domínio Atividades de Vida Diária correlacionou-se com a ALSFRS-R. Gradualmente, a diminuição da mobilidade leva a dificuldade na realização de atividades da vida diária e, às vezes, respiração, comunicação, sendo a alimentação também prejudicada. $3,16,17$

A sobrecarga do cuidador está associada a um maior comprometimento comportamental e físico do paciente com ELA, e a sentimentos mais depressivos do cuidador. ${ }^{18}$

Verificou-se nesta pesquisa que os cuidadores informais apresentam moderada sobrecarga de estresse. De um modo geral, os cuidadores experimentaram maior prevalência de problemas vinculados a Implicações na vida pessoal (diminuição do tempo disponível para si, refletindo na sua saúde e vida social, aumento do esforço físico, da sensação de redução da liberdade, e de alteração dos projetos de vida), e a Reações a exigências (aspectos que capacitam ou facilitam o cuidador a continuar a enfrentar os problemas decorrentes do desempenho desse papel). Esta maior sobrecarga vivenciada pelos cuidadores, possui uma relação significativa com os níveis de dependência funcional dos pacientes com ELA. ${ }^{19}$

A dimensão menos preocupante para os cuidadores avaliados, refere-se à sobrecarga financeira (dificuldades econômicas decorrentes à situação de doença do familiar e à incerteza quanto ao futuro). A preocupação com aspectos financeiros depende do estágio da doença, pacientes em estágio inicial aumentam a preocupação financeira, entretanto o avanço da doença e a sensação de proximidade da morte influencia, diminuindo o valor atribuído aos recursos financeiros. ${ }^{20}$

O apoio social funcional é um conceito subjetivo, que diz respeito às percepções do indivíduo sobre a sua própria disponibilidade de suporte. Os dados indicam que para a amostra estudada, há uma maior percepção da existência de pessoas em sua rede social que lhes amem (Apoio afetivo) e com quem podem ter momentos de prazer e relaxamento (Interação Social), e apoiar-Ihes com medidas práticas (Apoio Material).

Contudo, percebem menos a possibilidade de terem com quem contar para dividir suas preocupações e sentimentos (Apoio Emocional) e solicitar informações e ajuda (Informacional). Os benefícios de receber apoio informacional podem ser superestimados em cuidadores de pessoas com ELA, pois assumir tarefas de cuidar, especialmente nas fases avançadas da doença é complicado, visto que geralmente outros membros da família e amigos não estão familiarizados e instrumentalizados para oferecer suporte físico, em contrapartida o apoio emocional pode ser mais factível para aliviar a carga do cuidador. ${ }^{18}$

Cabe ressaltar que a MOS avalia a percepção da existência de apoio social, mas não sua efetividade nem quantidade. Desta forma, os dados demonstram que os participantes percebem mais o apoio afetivo, contudo, não apontam a quantidade de pessoas disponíveis para este apoio. Um maior apoio social pode lançar bases para uma melhor percepção de saúde, o que em conjunto diminui o estresse, prevenindo o desenvolvimento de depressão entre os cuidadores. ${ }^{21}$

Todavia, à escassez de pesquisas quantitativas neste tópico, que especifiquem os subtipos de apoio social percebidos impossibilita generalizações. ${ }^{18}$

As análises de correlações entre as subescalas da MOS e QUASI apresentaram-se negativas por conta da inversão das pontuações dos itens das três dimensões positivas do segundo instrumento.

O Apoio Afetivo correlacionou-se com a subescala Satisfação com o Papel e com o Familiar, que diz respeito a sentimentos e emoções positivas, aumento de autoestima e sentir-se mais próximo do familiar afetado, em detrimento do desempenho do papel de cuidador, e da relação afetiva que o mesmo estabelece com o sujeito alvo dos cuidados. Em nossa amostra todos os cuidadores apresentaram uma forte proximidade parental com o familiar com ELA. Esta 
proximidade e o tipo de relação afetiva existente entre o cuidador e o paciente anteriormente à doença, contribuem para o processo de inserção e adaptação do cuidador neste papel. $^{6}$

Os custos associados à ELA são maiores quando comparados a outras doenças neurológicas, indicando muitas vezes a necessidade de apoio financeiro para pacientes e familiares, pois custos indiretos substanciais podem ser impulsionados pela redução da renda dos pacientes e dos cuidadores informais. $^{22}$

A correlação do Apoio Material com a subescala Mecanismos de Eficácia e de Controle, demonstra que a disponibilidade de recursos financeiros pode aumentar os aspetos que capacitam ou facilitam o cuidador a enfrentar os problemas que advêm desta ocupação.

Apesar de suas necessidades, os cuidadores relutam em solicitar e aceitar apoio, reconhecendo suas próprias necessidades como secundárias às necessidades dos pacientes. ${ }^{23}$ A coesão familiar, a satisfação com a família e a qualidade da comunicação entre os familiares estão associados a níveis reduzidos de sobrecarga de cuidadores de pacientes neurológicos, enquanto o não envolvimento familiar está associado a uma maior sobrecarga. ${ }^{24}$

A correlação entre as subescalas de Suporte familiar e Interação social positiva, demonstram que o reconhecimento, apoio e envolvimento que os demais membros da família disponibilizam ao principal cuidador, pode influenciar positivamente em suas relações sociais. A ampliação das redes pessoais e comunitárias do cuidador auxilia na superação do isolamento, além de aprimorar os cuidados prestados ao familiar com ELA. ${ }^{25}$

A literatura evidencia que pacientes com ELA e seus cônjuges não diferem em relação aos níveis de percepção de solidão, o que reflete em preocupação com a adequação das relações sociais próximas. ${ }^{20} \mathrm{Um}$ corpo moderado de evidências demonstra que cuidadores, que experimentam sintomas depressivos, são mais propensos a apresentar alta sobrecarga. ${ }^{18}$

Apesar da escassez de estudos sobre o apoio social nos cuidadores dos pacientes com ELA, e de nossa pesquisa não ter utilizados avaliações específicas para mensuração de sintomas depressivos. Nossos achados sugerem, que o elevado suporte social foi uma variável moderadora na relação entre a sobrecarga de estresse percebido pelos cuidadores informais que compuseram nossa amostra.

\section{CONCLUSÕES}

Um serviço de reabilitação deve ser centrado na pessoa com ELA, na sua família e nos seus cuidadores, que experimentam uma série de reações emocionais devido à natureza progressiva da doença.

Este estudo explorou simultaneamente aspectos psicossociais importantes do paciente e do cuidador durante o acompanhamento de reabilitação, desenvolvendo uma abordagem centrada na pessoa e na família. Esses conhecimentos possibilitam a identificação de cuidadores em risco de sobrecarga e orientam o desenvolvimento de intervenções para diminuir esta percepção.
O estudo realizado apresenta limitações importantes quanto ao tamanho da amostra, que ao se apresentar em número reduzido, inviabiliza generalizações significativas a partir dos dados, já que as provas estatísticas requerem um tamanho amostral maior para garantir uma tendência, e uma distribuição representativa para grupos de pessoas, permitindo considerar os resultados encontrados apenas para a população em questão.

Todavia, embora os achados demonstrem uma realidade local, traz contribuições visto que, elucida indicadores importantes referentes aos efeitos da funcionalidade e da qualidade de vida do paciente com Esclerose Lateral Amiotrófica, na provisão de cuidados e na percepção sobre a sobrecarga e apoio social do cuidador.

Como recomendações para investigações futuras nesta mesma temática, salientamos a importância de cooperações entre Centros de Reabilitação, com o objetivo de aumentar o número amostral de participantes. Desejamos ainda, em estudos posteriores realizar um monitoramento longitudinal dos pacientes e seus familiares, com o intuito de acompanhar a relação entre o suporte social e a sobrecarga dos cuidadores, com a funcionalidade e qualidade de vida do paciente nos diferentes estágios de progressão da doença.

\section{REFERÊNCIAS}

1. Majmudar S, Wu J, Paganoni S. Rehabilitation in amyotrophic lateral sclerosis: why it matters. Muscle Nerve. 2014;50(1):4-13. Doi: https://doi.org/10.1002/mus.24202

2. Paganoni S, Karam C, Joyce N, Bedlack R, Carter GT. Comprehensive rehabilitative care across the spectrum of amyotrophic lateral sclerosis. NeuroRehabilitation. 2015;37(1):53-68. Doi: https://doi.org/10.3233/nre151240

3. Rosa Silva JP, Santiago Júnior JB, Santos EL, Carvalho FO, França Costa IMP, Mendonça DMF. Quality of life and functional independence in amyotrophic lateral sclerosis: A systematic review. Neurosci Biobehav Rev. 2020;111:111. Doi: https://doi.org/10.1016/j.neubiorev.2019.12.032

4. Sandstedt $P$, Littorin $S$, Cröde Widsell $G$, Johansson $S$, Gottberg K, Ytterberg C, et al. Caregiver experience, health-related quality of life and life satisfaction among informal caregivers to patients with amyotrophic lateral sclerosis: A cross-sectional study. J Clin Nurs. 2018;27(2324):4321-30. Doi: https://doi.org/10.1111/jocn.14593

5. Almeida LMS, Falcão IV, Carvalho TL. Evaluation of overloading on caregivers of people with Amyotrophic Lateral Sclerosis (ALS). Brazil J Occup Ther. 2017;25(3):585-93. Doi: http://dx.doi.org/10.4322/25268910.ctoA00871

6. Johnson S, Alonso B, Faulkner K, Roberts H, Monroe B, Lehman L, et al. Quality of life perspectives of people with amyotrophic lateral sclerosis and their caregivers. Am J Occup Ther.2017;71(3):7103190010p1-p7. Doi: https://doi.org/10.5014/ajot.2017.024828

7. Monteiro EA, Mazin SC, Dantas RAS. Questionário de Avaliação da Sobrecarga do Cuidador Informal: validação para o Brasil. Rev Bras Enferm. 2015;68(3):421-8. Doi: https://doi.org/10.1590/0034-7167.2015680307i 
8. Galvin M, Corr B, Madden C, Mays I, McQuillan R, Timonen $\mathrm{V}$, et al. Caregiving in ALS-a mixed methods approach to the study of burden. BMC Palliative Care. 2016;15(1):81. Doi: https://doi.org/10.1186/s12904-016-0153-0

9. Chen D, Guo X, Zheng Z, Wei Q, Song W, Cao B, et al. Depression and anxiety in amyotrophic lateral sclerosis: correlations between the distress of patients and caregivers. Muscle Nerve. 2015;51(3):353-7. Doi: https://doi.org/10.1002/mus.24325

10. Zanini DS, Verolla-Moura A, Queiroz IPdAR. Apoyo social: aspectos de la validez del constructo en estudiantes universitarios. Psicol Estud. 2009;14(1):195-202. Doi: https://doi.org/10.1590/S1413-73722009000100023

11. Associação Brasileira de Empresas de Pesquisa. Critério de Classificação Econômica Brasil. São Paulo: ABEP; 2015.

12. Guedes K, Pereira C, Pavan K, Valério BCO. Cross-cultural adaptation and validation of als Functional Rating ScaleRevised in Portuguese language. Arq Neuropsiquiatr. 2010;68(1):44-47. Doi: https://doi.org/10.1590/S0004282X2010000100010

13. Pavan K, Marangoni BE, Zinezzi MO, Schmidt KB, Oliveira $B C$, Buainain RP, et al. Validation of the Amyotrophic Lateral Sclerosis Assessment Questionnaire (ALSAQ-40) scale in the portuguese language. Arq Neuropsiquiatr. 2010;68(1):48-51. Doi: http://dx.doi.org/10.1590/S0004282X2010000100011

14. Navarro D, Foxcroft D. Learning statistics with jamovi: A tutorial for psychology students and other beginners (Version 0.70). 2019.2 Doi: http://dx.doi.org/10.24384/hgc3-7p15

15. Riviere $M$, Meininger $V$, Zeisser $P$, Munsat T. An analysis of extended survival in patients with amyotrophic lateral sclerosis treated with riluzole. Arch Neurol. 1998;55(4):526-8. Doi: https://doi.org/doi:10.1001/archneur.55.4.526

16. Felgoise SH, Zaccheo V, Duff J, Simmons Z. Verbal communication impacts quality of life in patients with amyotrophic lateral sclerosis. Amyotroph Lateral Scler Frontotemporal Degener. 2016;17(3-4):179-83. Doi: https://doi.org/10.3109/21678421.2015.1125499

17. Schultz J. Disease-modifying treatment of amyotrophic lateral sclerosis. Am J Manag Care. 2018;24(15 Suppl):S327-35.

18. Wit J, Bakker LA, van Groenestijn AC, van den Berg LH, Schröder CD, Visser-Meily JM, et al. Caregiver burden in amyotrophic lateral sclerosis: a systematic review. Palliat Med. 2018;32(1):231-45.

Doi: https://doi.org/10.1177/0269216317709965

19. Siciliano M, Santangelo G, Trojsi F, Di Somma C, Patrone $M$, Femiano $C$, et al. Coping strategies and psychological distress in caregivers of patients with Amyotrophic Lateral Sclerosis (ALS). Amyotroph Lateral Scler Frontotemporal Degener. 2017;18(5-6):367-377. Doi: https://doi.org/10.1080/21678421.2017.1285316

20. Segerstrom SC, Kasarskis EJ, Fardo DW, Westgate PM. Socioemotional selectivity and psychological health in amyotrophic lateral sclerosis patients and caregivers: a longitudinal, dyadic analysis. Psychol Health. 2019;34(10):1179-1195. Doi:
21. Aoun SM, Bentley B, Funk L, Toye C, Grande G, Stajduhar KJ. A 10-year literature review of family caregiving for motor neurone disease: moving from caregiver burden studies to palliative care interventions. Palliat Med. 2013;27(5):437-446.

Doi: https://doi.org/10.1177/0269216312455729

22. Gladman M, Zinman L. The economic impact of amyotrophic lateral sclerosis: a systematic review. Expert Rev Pharmacoecon Outcomes Res. 2015;15(3):439-50. Doi: https://doi.org/10.1586/14737167.2015.1039941

23. Wit J, Schröder CD, El Mecky J, Beelen A, van den Berg LH, Visser-Meily JM. Support needs of caregivers of patients with amyotrophic lateral sclerosis: a qualitative study. Palliat Support Care. 2018;5:1-7. Doi: https://doi.org/10.1017/S1478951517001213

24. Tramonti F, Bonfiglio L, Bongioanni P, Belviso C, Fanciullacci C, Rossi B, et al. Caregiver burden and family functioning in different neurological diseases. Psychol Health Med. 2019;24(1):27-34. Doi: https://doi.org/10.1080/13548506.2018.1510131

25. Cipolletta S, Gammino GR, Francescon P, Palmieri A. Mutual support groups for family caregivers of people with amyotrophic lateral sclerosis in Italy: A pilot study. Health Soc Care Community. 2018;26(4):556-63. Doi: https://doi.org/10.1111/hsc.12558 